\title{
POSTER: Towards Architecture and OS-Independent Malware Detection via Memory Forensics
}

\author{
Rachel Petrik ${ }^{*}$, Berat Arik ${ }^{\dagger}$, Jared M. Smith ${ }^{\dagger} \S$ \\ ${ }^{*}$ University of Kentucky, ${ }^{\dagger}$ University of Tennessee, Knoxville, ${ }^{\S}$ Oak Ridge National Lab
}

\begin{abstract}
In this work, we take a fundamentally different approach to the problem of analyzing a device for compromises via malware; our approach is OS and instruction architecture independent and relies only on having the raw binary data extracted from the memory dump of a device. Our system leverages a multi-hundred TB dataset of both compromised host memory dumps extracted from the MalRec dataset [8] and the first known dataset of benign host memory dumps running normal, non-compromised software. After an average of 30 to 45 seconds of pre-processing on a single memory dump, our system leverages both traditional machine learning and deep learning algorithms to achieve an average of $98 \%$ accuracy of detecting a compromised host.
\end{abstract}

\section{CCS CONCEPTS}

- Security and privacy $\rightarrow$ Malware and its mitigation; Intrusion detection systems; • Computing methodologies $\rightarrow$ Supervised learning;

\section{KEYWORDS}

Digital Forensics; Memory Forensics; Machine Learning; Deep Learning; CNN; LSTM

\section{INTRODUCTION AND BACKGROUND}

According to a recent study of 477 companies, the mean time to discover a breach is 197 days, with costs to remediate these breaches exceeding \$3.5 million on average [5]. Having an effective Digital Forensics and Incident Response (DFIR) capability remains one of the most effective ways to both prevent and respond to modern breaches. However, with the proliferation of intrusion detection systems and security products on the market, DFIR professionals and security analysts must monitor an increasing amounts of tickets and alerts from ever-growing infrastructure. To that end, one of the most effective and widely-used methods to investigate hosts and their operating systems (OS) for breaches relies on memory forensics, or the examination of a device's volatile RAM. Among the binary data in RAM are the names and metadata of running and recently exited processes, system kernel modules and libraries, executable source code, file information, network information, registry keys, and more. Although tools such as Volatility and Rekall $[3,12]$

Permission to make digital or hard copies of part or all of this work for personal or classroom use is granted without fee provided that copies are not made or distributed for profit or commercial advantage and that copies bear this notice and the full citation on the first page. Copyrights for third-party components of this work must be honored.

For all other uses, contact the owner/author(s).

CCS '18, October 15-19, 2018, Toronto, ON, Canada

(C) 2018 Copyright held by the owner/author(s)

ACM ISBN 978-1-4503-5693-0/18/10.

https://doi.org/10.1145/3243734.3278527 are able to successfully extract the OS-specific data and potential indicators of compromise (IOCs) from memory, they require experts to build and maintain the systems, requiring an addition of new profiles for every new OS and version. Furthermore, any artifacts found in memory must be verified by an expert with relevant OS and architecture-specific knowledge, even when these artifacts may not actually represent a compromise.

By capitalizing on the ongoing breakthroughs in deep learning [9], as well as more traditional machine learning techniques, we present a system to detect malware-compromised hosts via captured memory snapshots alone. By not relying on OS-specific information, we aim to reduce the amount of time an analyst must focus on a host by flagging potentially compromised hosts with only a memory snapshot as input. Most importantly, our system could be deployed in any operational environment where a memory snapshot can be ingested from connected hosts, without relying on endpoint IDSes that require OS and domain-specific IOCs. Systems such as Akatosh by Smith et al. [10] provide the ability to scalably ingest memory images from hosts, as well as others such as Endcase [2]. Our system utilizes a multi-hundred TB dataset of both compromised host memory snapshots extracted from the MalRec dataset [8] and the first known dataset of benign host memory snapshots running normal, non-compromised software. After an average of 30-45 seconds of pre-processing on a single memory snapshot, our system leverages both traditional machine learning and deep learning algorithms to achieve an average of $98 \%$ accuracy of detecting a compromised host, with over 3,000 samples for our Convolutional Neural Network, and over 9,000 samples for our traditional machine learning models.

\section{DATASETS}

\subsection{Compromised (Malicious) Snapshots}

We employed the MalRec dataset [8] created by Georgia Tech over the course of two years. With over $66,000+$ malware recordings from Windows 7 32-bit machines using the QEMU virtualization software, our infrastructure extracts 1 GB memory snapshots at $0 \%$ of the QEMU recording, a random percent between 5\% and 95\%, and finally a snapshot at $99 \%$ of the execution of the recording. Given that the malware for a particular recording executes shortly after the beginning of the recording, we are able to use the $0 \%$ as a baseline snapshot. In practice, we believe the random percentage capture models the real-world best, where an operator cannot ensure a memory snapshot is captured after malware has completely executed in memory. Finally, these recordings contain traces of memory of normal programs, depending on what the malware calls into and passes input to; this could be PDF viewers, the commandline, browsers, and more pivot points for the malware to infect the machine. 


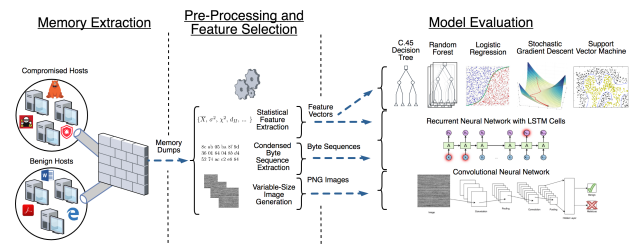

Figure 1: High-Level Architecture of the memory extraction, pre-processing and feature selection, and model evaluation.

\subsection{Benign Snapshots}

Next, we created a dataset of benign host memory snapshots running normal, non-compromised software, including software that executes in many of the malicious snapshots. We do not claim our dataset is completely robust, and work remains to build additional representations of "normal" state represented by volatile RAM. To generate this dataset, we leverage VirtualBox and Python to instrument a running VM. After ensuring the VM uses the same size snapshots as MalRec (1 GB), each sample generated includes the execution of between 1 and 9 programs from a list of non-malicious programs that a user might run such as Google Chrome, PDF Viewers, command-line consoles, Spotify, Slack, and Skype. Once the programs launched, we waited $20+\left(5 \times n_{p}\right)$ seconds, where $n_{p}$ is the number of programs ran. After this time, a benign snapshot is extracted from memory after ample time has passed for the chosen programs to open. By generating samples in parallel to the separate malicious environment, we formed our benign memory snapshot dataset. Additional work should be done to done building 'profiles' of users in order to have distinct sets of benign snapshots, centered around different demographics and job functions of users in practice. Furthermore, the time between program execution and memory snapshot collection should be varied further, while user input should be passed automatically to the executed programs to further simulate real users. Figure 1 below shows the dataset collection phase of our system, as well as highlights the next phases.

\begin{tabular}{|c|c|c|}
\hline \multicolumn{3}{|c|}{ Statistical Features } \\
\hline Min/Max & $\begin{array}{c}\text { Geometric, Harmonic, Standard Error, } \\
\text { Arithmetic Mean }\end{array}$ & $\begin{array}{c}\text { Skew, Kurtosis, } \\
\text { Variance, Standard } \\
\text { Deviation }\end{array}$ \\
\hline \multirow{2}{*}{ Entropy } & $\begin{array}{c}\text { Hamming, Energy, Eucliden, } \\
\text { Wasserstein, Bray-Curtis Minkowski } \\
\text { Distance }^{*}\end{array}$ & $\begin{array}{c}\text { Jaccard-Needham } \\
\text { Dissimilarity }\end{array}$ \\
\hline Chi-Square Test & P-adic Valuation $^{*}$ & Shapiro-Wilk Test \\
\hline
\end{tabular}

Table 1: Total of 43 domain-unaware features extracted from memory snapshots. Starred features were extracted for several different numerical parameters.

\section{PRE-PROCESSING \& FEATURE SELECTION}

With the first phase of snapshot extraction completed, we investigated various approaches for representing the memory snapshots, without relying on domain-knowledge of the OS. Challenges arose with many aspects of this problem, particularly with how to store the data generated earlier, and more importantly, how to derive statistical and numerical features from binary data (the snapshot) that are comprehensive enough to uniquely identify each class of snapshots, benign or malicious. Beyond extracting features in vectors per sample, we generated images for use in a Convolutional Neural Network and byte sequences for a Recurrent Neural
Network with LSTM cells. Overall, we attempted to rid ourselves of the problem of expert knowledge by leveraging modern machine learning to learn the data itself after our system provides adequate feature representations.

Shown in Figure 1, for every malicious or benign snapshot, we applied two additional transformations beyond using the full snapshot before generating representations: (1) we removed the null-bytes in the images, reducing the snapshot size by over half, and (2) applied a recent system called MemScrimper [1] to deduplicate the snapshots based on a reference snapshot (which is the $0 \%$ image for the malicious snapshots, and the clean state of the VM for the benign snapshots). After this, we extracted images of various sizes $(1000 \times 1000,2500 \times 2500$, and $5000 \times 5000)$ by condensing the entire binary matrix, which is done by averaging every $n$ bytes, where $n$ depends on the original size of the snapshot. A sample of these images is shown in architecture diagram in Figure 1. We also generated condensed raw byte sequences in a similar manner. Finally, we applied a plethora of statistical and numerical methods to each snapshot representation to extract 43 features, including features for prime-number metrics, distance metrics, statistical metrics such as standard deviation, and even probability tests. These features are shown in Table 1. For all of these feature selection methods and running on a single core of a Linux-Based machine with a 2.5 $\mathrm{GHz}$ Intel Xeon processor, we show in Figure 2 that the average time to complete processing of one snapshot is less than 35 seconds for feature vector extraction. Furthermore, the average time for generating all images or extracting the byte sequences is less than 10 seconds. These methods were written in Python with some metrics using Numpy or Scipy; therefore, so significant speedups could potentially be gained when implemented all in $\mathrm{C}$ or $\mathrm{C}++$.
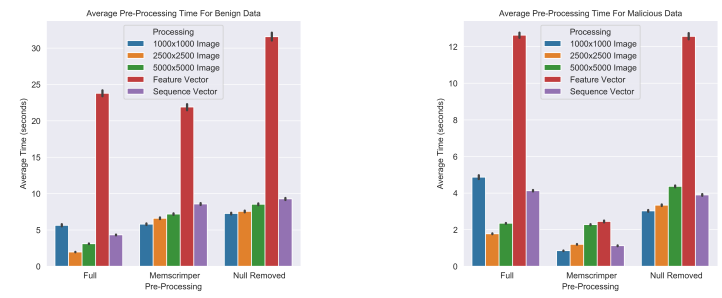

Figure 2: Average Pre-Processing Time for Benign (left) and Malicious (right) Memory Snapshots

\section{TRADITIONAL MACHINE LEARNING}

For our approach to detecting compromises within the snapshots using traditional machine learning, we employed a C.45 Decision Tree, a standard Logistic Regression model, a Random Forest model, a Stochastic Gradient Descent model, and a Support Vector Classifier. All machine learning methods used Scikit-Learn and an Ubuntu Linux 12-core $2.5 \mathrm{GHz}$ Intel Xeon system with $32 \mathrm{~GB}$ of RAM. For each model, we conducted a probabilistic grid search over all available hyper-parameters to tune the model, and when testing we used cross-validation and a non-overlapping $75 \% / 25 \%$ train/test split.

Overall, our traditional machine learning methods recorded an average of $98 \%$ accuracy of detecting a compromised host with 9,000 samples. We found the feature vectors generated from the memory snapshots that had been de-duplicated using MemScrimper performed the best across all traditional machine learning algorithms. 


\begin{tabular}{|l||l|l|l|l|}
\hline \multicolumn{5}{|c|}{ Traditional ML Results: Benign vs. Assorted\%, Memscrimper } \\
\hline ML Algorithm & Mean Test Accuracy & Mean Fit Time (s) & Mean Test Time (s) & FPR \\
\hline Decision Tree & 0.995851 & 0.017205 & 0.001449 & $0.002667 \%$ \\
Logistic Regression & 0.994814 & 0.081668 & 0.0247176 & $0.002667 \%$ \\
Random Forest & 0.996507 & 0.781202 & 0.002633 & $0.002667 \%$ \\
SGD & 0.991555 & 0.033968 & 0.012932 & $0.001778 \%$ \\
SVM & 0.994518 & 0.125464 & 0.013279 & $0.002667 \%$ \\
\hline
\end{tabular}

Table 2: Results obtained from running 9,000 de-duplicated memory captures through several different traditional machine learning algorithms with a train/test split of $25 \%$.

Notably, the false positive rate of this particular setting was also less than $0.003 \%$ on average, indicating that our system would add only a small fraction of false alerts to an analyst's dashboard in an environment with similar hosts. The summary of results from de-duplicated snapshots can be found in Table 2. Due to space constraints, we leave out the results for the full and null-byte-removed snapshots for both the random or assorted percentage and the $99 \%$ snapshots, though we still found the accuracy was above $95 \%$ on average but with slightly higher false positive rates averaging $0.1 \%$.

\section{DEEP LEARNING APPROACH}

Our primary deep learning approach was through the use of a Convolutional Neural Network (CNN), trained using various sized images from the different pre-processing methods outlined earlier. Our model was composed of multiple layers, including pooling and fully connected layers. We used a similar probabilistic grid search as before, except allowing both the hyper-parameters and model layers to be actively tuned in response to better performance. Under cross-validation and $75 \% / 25 \%$ train/test split, we show our results demonstrating the accuracy in Figure 3. For over 3,000 samples evenly balanced between benign and malicious images, our best $\mathrm{CNN}$ model finished with an accuracy of $98 \%$ when using the largest size of images generated from a non-condensed memory snapshot. Because of our low sample size, we also include rigorous data augmentation aligned with the best practices in the Keras deep learning library, which increases the generality of the model when training. Though the full image indicates strong accuracy, worse performance was found for the null-byte-removed and MemScrimper images. Regardless, our future work includes training and testing on more samples, which our experiment infrastructure continues to extract from the benign and malicious host testbeds.
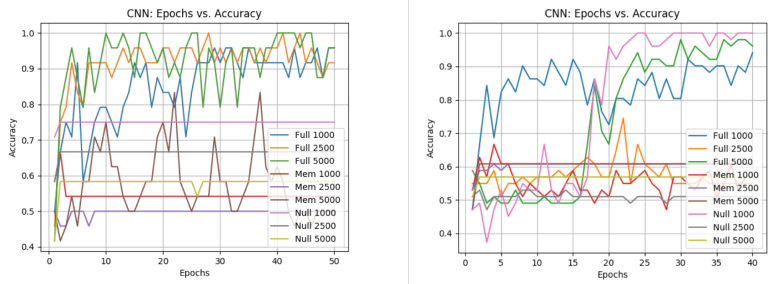

Figure 3: CNN Accuracy over 50 Epochs for Random Percent Malicious Snapshot (left) versus 99\% Malicious Snapshot (right)

\section{RELATED WORK}

Most closely related to our analysis of memory snapshots as images is work originally by Nataraj et al. and Kamundala et al. [6, 7]. Nataraj focused on representing malware executables as images, which was then followed by extensions to the original study applying a CNN-based strategy to other forms of malware, including datasets of Android samples [4]. Our work differs significantly from these systems once we consider the entire memory snapshot of a device containing a malware's trail in volatile memory.

With a similar motivation and a benign dataset developed concurrently but independently of ours, the DeepMem system by Song et al. [11] to appear in CCS 2018 leverages graph-based deep learning to locate domain-specific data in memory snapshots. Beyond the similar datasets, our work diverges in that our approach requires no specific representation of the data in the snapshot, rather leveraging an array of statistical and numerical features independent of any inherent structure.

\section{CONCLUSION}

In this paper, we presented to the best of our knowledge, the first steps towards an OS and architecture-independent malware detection system, exploiting several pre-processing techniques, domainunaware feature selection, and a suite of machine learning algorithms. We built a system for capturing memory snapshots compromised by malware, as well as a novel benign memory snapshot dataset. With our datasets, we ultimately evaluated the feasibility of our approach by testing against 3,000+ samples balanced between benign and malicious snapshots and with cross-validation. Our system achieved over $98 \%$ accuracy with low false-positive-rate for most traditional machine learning algorithms and saw similar results for a more complex, image-based $\mathrm{CNN}$ architecture.

\section{ACKNOWLEDGEMENTS}

The authors would like to thank Parker Diamond for contributing the early ideas that started this work, Jason Laska for providing essential technical review of the manuscript, and Kaleigh Veca for extensive revision that lead to greater clarity of the story. Oak Ridge National Laboratory is managed by UT-Battelle, LLC for the US Department of Energy under contract DE-AC05-00OR22725.

\section{REFERENCES}

[1] Michael Brengel and Christian Rossow. 2018. MemScrimper - Time- and SpaceEfficient Storage of Malware Sandbox Memory Dumps. DIMVA (2018).

[2] Endcase. 2018. Endcase Forensics. https://www.guidancesoftware.com/ encase-forensic

[3] Google. 2018. Rekall Forensics. http://rekall-forensic.com

[4] TT Huang and Kao HY. 2017. R2-D2: Color-Inspired Convolutional Neural Network (CNN)-Based Android Malware Detection. https://arxiv.org/abs/1705. 04448. arxiv.org (2017).

[5] IBM, Ponemon Institute. 2018. Cost of Data Breach Study. https://www.ibm. com/security/data-breach

[6] Espoir K Kamundala and Chang Hoon Kim. 2018. CNN Model to Classify Malware Using Image Features. KIISE Transactions on Computing Practices 24, 5 (2018), 256-261.

[7] Lakshmanan Nataraj, Sreejith Karthikeyan, Gregoire Jacob, and BS Manjunath. 2011. Malware Images: Visualization and Automatic Classification. In Proceedings of the 8th International Symposium on Visualization for Cyber Security. ACM, 4.

[8] Giorgio Severi, Tim Leek, and Brendan Dolan-Gavitt. 2018. Malrec - Compact Full-Trace Malware Recording for Retrospective Deep Analysis. DIMVA (2018).

[9] Karen Simonyan and Andrew Zisserman. 2015. Very Deep Convolutional Networks for Large-Scale Image Recognition. International Conference on Learning Representations (ICLR) (2015).

[10] Jared M Smith, Elliot Greenlee, and Aaron Ferber. 2017. DEMO: Akatosh Automated Cyber Incident Verification and Impact Analysis. CCS (2017), 24632465.

[11] Song, Wei, Yin, Heng, Liu, Chang, and Song, Dawn. 2018. DeepMem: Learning Graph Neural Network Models for Fast and Robust Memory Forensic Analysis. ACM Conference on Computer and Communications Security (CCS) (2018).

[12] The Volatility Foundation. 2018. Volatility Foundation. https:/www. volatilityfoundation.org 